\title{
¿Por qué es importante establecer una rúbrica de evaluación? El caso del curso Clínica de Exodoncia y Cirugía
}

\author{
Why is important to establish an evaluation rubric? \\ The case of the curse Clinic of Exodontia and Surgery
}

\author{
Patricia Hernández-Rivera \\ Docente Facultad de Odontología \\ Universidad de Costa Rica \\ San José, Costa Rica \\ patricia.hernandezrivera@ucr.ac.cr
}

Recibido: 08-VI-2011 • Aceptado: 22-VII-2011 • Corregido: 25-VI-2012

\begin{abstract}
Resumen: En la actualidad los procesos evaluativos han cobrado importancia, pues se requieren instrumentos objetivos que ofrezcan un medio para valorar las competencias logradas por el estudiantado, en determinado proceso de enseñanza-aprendizaje. Las rúbricas de evaluación ofrecen un mecanismo objetivo, que estandariza los indicadores a valorar, según el rendimiento mostrado por el estudiantado. Además, le permite al personal docente orientar y retroalimentar al alumnado, quienes también podrán utilizar el instrumento para autoevaluarse y prepararse de antemano, de acuerdo con las especificaciones descritas en la rúbrica. El objetivo del presente trabajo es elaborar una rúbrica de evaluación para el curso de Clínica de Exodoncia y Cirugía. Dicho instrumento se realizó considerando las opiniones del $40 \%$ del profesorado a cargo del curso, por medio de una entrevista semi-estructurada. Esta rúbrica ofrece una herramienta que estandariza el proceso de evaluación de las "presentaciones de caso" y permite que el equipo docente pueda retroalimentar al estudiantado y favorece la interacción docente-estudiante.
\end{abstract}

Palabras clave: Rúbrica, evaluación, evaluación clínicacognición, estilos de aprendizaje.

\section{Introducción}

En la actualidad las instituciones educativas han tenido que readecuar sus programas, según las exigencias de una sociedad globalizada, que requiere de profesionales integrales, que cumplan con diversas competencias que no están directamente relacionadas con la carrera profesional, pero que son fundamentales. De manera que tengan la capacidad para resolver problemas, de trabajar en forma colaborativa, de tener la habilidad del aprendizaje continuo, de utilizar adecuadamente las tecnologías de la información y la comunicación, y entre otras características, que mejoren el desempeño laboral (Altbach, Reisberg y Rumbley, 2009). Por ello, el personal docente debe establecer las competencias deseables en el estudiantado, como parte 


\begin{abstract}
Nowadays the evaluation process has gained importance, because teachers need an objective instrument that value the competencies achieved by students, during the learning-teaching process. The evaluation rubrics offer an objective way that standardized the indicators to be valued, against the performance shown by the student. Also the rubrics help teachers to guide and to feedback students, who can use it to self-evaluate and prepare the assignments according to the specifications in the rubric. The object of this work was to make an evaluation rubric for the course "Clinic of Exodontia and surgery". This instrument was constructed taking into consideration the opinions of the $40 \%$ of the teachers of the course, gathered in a semi-structured interview. These rubrics standardized the evaluation process for the "Case presentation" and help teachers to feedback students and also improve the interaction between teacher-student.
\end{abstract}

Keywords: rubric, evaluation, clinical evaluation. de las obligaciones curriculares, debido a que el objetivo es lograr que los conocimientos teóricos, los procedimientos y las actitudes se confabulen en el desarrollo de una formación integral que consiga profesionales competentes. Por lo tanto, se requiere que el profesorado exponga situaciones prácticas que asemejen casos reales, de manera que el estudiantado adquiera la destreza y la adaptación de un determinado contexto para resolver problemas a los que en su futuro profesional se enfrentará (Tejada, 2005; Curran, Casimiro, Banfield, Hall, Lackie, Simmons, et al., 2009).

Los argumentos esbozados anteriormente acrisolan la relevancia que tienen los procesos de evaluación y aunque, tanto profesores como estudiantes están habituados a éstos, se les debe concientizar que la evaluación es un proceso sistemático, tendiente a juzgar el aprendizaje desarrollado por el alumnado en determinada actividad educativa, según parámetros establecidos (Foss, 2009).

Sin embargo, la evaluación debe convertirse en parte del proceso de enseñanzaaprendizaje, permitiendo que el personal docente y el estudiantado participe activamente de este, desarrollando un espacio de retroalimentación y análisis del mejoramiento académico a lo largo del curso lectivo (Hernández y Moreno, 2007).

Debido a la importancia que tiene la evaluación en el crecimiento académico del estudiantado; en el presente estudio se plantea una rúbrica que permite evaluar las "presentaciones de caso", las cuales son actividades educativas que le permiten al estudiantado resolver el manejo de un paciente real. En este estudio se enfocará en las "presentaciones de caso" realizadas por los estudiantes de quinto año, en la Clínica de Exodoncia y Cirugía de la Facultad de Odontología, de la Universidad de Costa Rica. Para cumplir con lo expuesto se plantearon dos objetivos específicos: a) Determinar las competencias que deberían desarrollar los estudiantes durante la Clínica de Exodoncia y Cirugía, en quinto 
año de carrera, y b) Realizar una rúbrica para evaluar las "presentaciones de caso" que realizan los estudiantes en esa Clínica.

\section{Referente teórico}

Existen tres tipos de evaluaciones según el indicador utilizado: las evaluaciones según el agente, por normotipo y según la función. Estas últimas se pueden subdividir en diagnósticas, formativas $\mathrm{y}$ sumativas, dependiendo del objetivo que se desea lograr o el periodo en el que se desarrollen (Santamaría, 2005). Las evaluaciones diagnósticas se realizan al inicio de cualquier actividad educativa y tienen el propósito de determinar los conocimientos previos que poseen los estudiantes, de manera que el personal docente conozca los conceptos que tiene el alumnado y sobre esa base pueda iniciar el desarrollo de la dinámica educativa. Otro tipo es la formativa, la cual se realiza durante el proceso de enseñanza-aprendizaje, con el objetivo de que el estudiantado pueda analizar su propio avance en el proceso educativo; permitiéndole aprender de sus propios errores y con ello, mejorar sus técnicas de estudio para lograr resultados exitosos. Además, el profesorado puede examinar los resultados del alumnado y si fuera el caso, replantear la estrategia didáctica y así obtener un mejor entendimiento de la materia tratada. Finalmente, están las evaluaciones sumativas, que son las más utilizadas y tienen como propósito medir o calificar el grado de aprendizaje logrado al finalizar una actividad educativa; su aplicación más común es determinar la aprobación o reprobación de los cursos (Valenzuela, 2004; Morales, 2010; Maroto, 2010).

Convencionalmente se han elaborado evaluaciones sumativas según el paradigma cuantitativo, asignándole un valor numérico a los aspectos descriptivos, de acuerdo al desempeño demostrado por el estudiantado. Aunque existen diversos factores que no pueden contabilizarse. Por ello, en la actualidad se buscan razonamientos cualitativos de evaluación, que valoren de forma holística el proceso educativo, considerando el cumplimiento de los objetivos estipulados y la metodología; lo que evita basarse exclusivamente en los resultados cuantitativos de un examen dejando de lado la evaluación global del proceso de enseñanza-aprendizaje (Hernández y Moreno, 2007).

Las evaluaciones cualitativas se caracterizan por el diseño de escalas nominales o la categorización por jerarquías. Esa información desprendida de este tipo de evaluaciones transforma al profesorado en orientador del proceso de enseñanza y genera relaciones horizontales entre el personal docente y el estudiantado, lo que promueve un ambiente de motivación hacia el mejoramiento académico (Hernández y Moreno, 2007).

En este contexto, las rúbricas de evaluación organizan un conjunto de criterios pre-establecidos, para valorar conocimientos, capacidades y actitudes de una actividad educativa. En general se presentan en forma de matriz, donde la primera columna del eje vertical especifica el parámetro que será evaluado y en el eje horizontal se registran los diferentes niveles de desempeño, lo que permite asignar un mayor o menor puntaje, según lo demostrado por el alumnado. En las siguientes columnas se hace una breve descripción de acuerdo al grado de cumplimiento del criterio expuesto en la primera columna (Kan, 2007; Martínez-Rojas, 2008). Razón por la cual, las rúbricas brindan una evaluación cualitativa, pero a su vez, se le puede otorgar un puntaje cuantitativo según el nivel demostrado por el estudiantado (MartínezRojas, 2008).

Además, evalúan no solo el resultado, sino el proceso de enseñanza-aprendizaje, pues permite analizar otros aspectos académicos como la metodología e inclusive el rol del docente. Además, crea un espacio para la retroalimentación, en el cual el personal 
docente y el estudiantado analizan las fortalezas y las debilidades demostradas, así como posibles recomendaciones, las cuales podrán ser re-evaluadas y valorar el mejoramiento en el rendimiento. Por otro lado, las rúbricas permiten que el proceso de evaluación sea más objetivo; pues el alumnado conoce con anticipación los elementos, parámetros y la escala de calificación con la cual será evaluado (Santamaría, 2005; Martínez-Rojas, 2008; Reeves y Stanford, 2009).

El análisis de los resultados que demuestran las rúbricas, le brindan al personal docente información sobre la efectividad de las técnicas didácticas empleadas durante las actividades educativas. Además, permiten que cada curso y el propio proceso de evaluación, puedan ser analizados por pares externos, lo que obviamente se refleja en ese continuo proceso de mejoramiento, lo cual es la meta de la educación superior (Martínez-Rojas, 2008; Reeves y Stanford, 2009).

El proceso para crear una rúbrica de evaluación se inicia identificando los objetivos de aprendizaje del curso, que representan las competencias que deberá cumplir el estudiantado al culminar el ciclo lectivo (Curran et al., 2009). Por lo tanto, es imprescindible que esos objetivos estén bien redactados y en concordancia con el grado de aprendizaje que se desea desarrolle el estudiantado al finalizar el curso en cuestión (Valenzuela, 2004). Posteriormente, se seleccionan los criterios de evaluación y se diseña una escala que valora el rendimiento académico en cada uno de los criterios consignados en la evaluación (Curran et al., 2009).

En el caso de la carrera de Licenciatura en Odontología, de la Universidad de Costa Rica, las evaluaciones deben responder al perfil profesional determinado, según las políticas de la Facultad y de esta forma el estudiantado aprueba o reprueba la materia, de acuerdo con las competencias demostradas durante el curso (Maroto, 2010).
Específicamente para el quinto año de la carrera, se debe cursar la Clínica de Exodoncia y Cirugía; en la cual, como parte de las actividades académicas, el estudiantado debe realizar "presentaciones de caso de cirugía", en las cuales deben abordar el estudio del (la) paciente, con el consiguiente análisis, según la patología que pudiese presentar; pues, de acuerdo con el análisis desarrollado, deben plantear la intervención quirúrgica que requiere esa persona. Estas presentaciones se efectúan previamente a la intervención quirúrgica, la cual es realizada por el personal académico, a manera demostrativa para ese(a) estudiante, aprovechando la experiencia para explicar los diversos procedimientos. Por esta razón, es importante incorporar una rúbrica de evaluación, para esa presentación de caso, que permita determinar de manera objetiva, el desempeño clínico del estudiantado en el área de Exodoncia y Cirugía.

Además, en dicha clínica el personal académico está conformado por 15 docentes, que tienen a su cargo la evaluación de un promedio de 60 estudiantes por curso, repartidos en los diferentes turnos clínicos. Por lo cual, su labor docente es prácticamente tutorial e incluye la evaluación de cada uno de los estudiantes a su cargo; con el agravante que no se aplican criterios semejantes en la evaluación. Además, el proceso de retroalimentación hacia el alumnado se torna deficiente y un tanto subjetivo, lo que va en detrimento del desarrollo de competencias en el área de Exodoncia y Cirugía. Por tal razón, debe establecerse un procedimiento estandarizado que asegure la uniformidad de criterios de evaluación, para que sea confiable y válida para todo el estudiantado involucrado.

Los argumentos anteriores justifican la necesidad de establecer una rúbrica de evaluación para esta sección, que asegure una metodología objetiva, que pueda ser utilizada de manera eficaz por la totalidad del equipo docente de esta sección y que a su vez, brinde una retroalimentación al 
estudiantado, para transformar sus debilidades en cualidades competentes y pertinentes, para su desarrollo profesional.

Por otro lado, la Facultad se comprometió a un proceso de mejoramiento de la calidad, tras obtener la acreditación ante el Sistema Nacional de Acreditación de la Educación Superior (SINAES), en el año 2009. Este proceso de mejoramiento sigue vigente y el compromiso adquirido incluye mejorar la evaluación en las diversas materias, lo cual fue una de las debilidades que señaló el alumnado en la autoevaluación realizada previo a la acreditación; por lo tanto, la convierte en uno de los imperativos que deben solventarse (Filloy y Howard, 2009). Esta situación confirma que el desarrollo de rúbricas de evaluación, es fundamental en el proceso de evaluación pues son técnicas evaluativas que tratan de aminorar la subjetividad, a fin de asegurar la calidad de la educación brindada.

\section{Metodología}

La primera fase de la construcción de la rúbrica de evaluación se enfocó en las "presentaciones de caso de cirugía", las cuales forman parte de los objetivos del curso de Clínica de Exodoncia y Cirugía. Para ello, se entrevistó a 6 personas (40\%) que forman parte del profesorado a cargo de la sección, utilizando preguntas semiestructuradas; lo que permitió determinar las competencias, que el personal docente de la sección consideró que debe cumplir el estudiantado en esas presentaciones.

La segunda fase consistió en agrupar los criterios expuestos por el equipo docente según categorías, tomando en cuenta las sugerencias que realizaron a la rúbrica de evaluación. Posteriormente, se realizó como fase final, el análisis y la construcción de las características para determinar los niveles de la rúbrica, y de esta forma, poder determinar el rendimiento académico del estudiantado.
A inicios del primer semestre del año 2011, al empezar el ciclo lectivo se entregó una rúbrica de la evaluación de las "presentaciones de caso de cirugía”, junto con el programa de la clínica, con el fin de explicarle al alumnado la forma en que iban a ser evaluados y que conocieran los rubros que se les iba a calificar.

\section{Resultados y su análisis}

Las competencias que deben evaluarse en el estudiantado matriculado en el curso de Clínica de Exodoncia y Cirugía, fueron descritas por el personal académico de la sección y agrupadas según los conocimientos y las actitudes que el alumnado debe desarrollar como producto del curso. Con respecto a los conocimientos, sobresalieron los temas de Patología sistémica, Farmacoterapia, Anatomía de la zona quirúrgica, Anestesia y Diagnóstico patológico; lo cual concuerda con los objetivos específicos para este curso. En lo referente al manejo del paciente con compromiso sistémico, es importante que el estudiantado comprenda la patología sistémica de fondo que presenta el o la paciente, los diversos métodos diagnósticos, los parámetros bioquímicos y hematológicos suministrados por el laboratorio clínico, así como el tratamiento que está tomando, las posibles interacciones medicamentosas de estos con los fármacos antimicrobianos, analgésicos y anestésicos locales. También, es importante que el educando analice las consideraciones durante la intervención quirúrgica, según la patología sistémica del paciente. Este tópico se relaciona estrechamente con el de anestesia, pues es importante que el alumnado conozca las diferentes soluciones anestésicas, el contenido de cada ampolla del anestésico, que en el argot odontológico se le denomina "cartucho"; por consiguiente, es importante también, que el estudiantado conozca la dosificación, el mecanismo de acción del anestésico en cuestión y las 
diferentes técnicas básicas de anestesia intraoral, según la cirugía que se realizará. Debido a que parte del objetivo del curso es que el estudiantado demuestre los conocimientos teóricos de los cursos de Anestesia y Exodoncia.

Otro elemento fundamental por evaluar, es el conocimiento sobre la anatomía del área donde se realiza la cirugía, lo que incluye el reconocimiento de estructuras anatómicas importantes y las implicaciones que estas representan. Adicionalmente, el estudiantado deberá llevar a cabo el proceso diagnóstico de acuerdo con el tipo de cirugía que se realice, sea esta de piezas incluidas (criptodontectomías) o de tejidos blandos; en el caso de piezas incluidas deberá clasificar la tercera molar, utilizando la Clasificación de Pell - Gregory, la Clasificación de Winter y realizar el análisis radiográfico correspondiente. En las cirugías de tejidos blandos deberá elaborar un diagnóstico diferencial y las descripciones de las posibles patologías. También, deberá ser capaz de describir el procedimiento quirúrgico que se llevará a cabo e indicar el instrumental y los materiales que se utilizarán. Además, deberá saber resolver las posibles urgencias inmediatas que se pudiesen presentar; predecir posibles complicaciones postquirúrgicas según el tratamiento efectuado y la forma correcta de tratarlas. En el tema de farmacoterapia, deberá determinar los medicamentos que le recetará al paciente, según su compromiso sistémico y el tratamiento quirúrgico. Así como conocer la posología y las razones por las cuales está indicando el fármaco.

En este apartado también se recalcó la importancia de asignar un tema teórico, de manera que el educando investigue, realice un análisis crítico de la información y pueda explicar lo comprendido. Esto con el objetivo de desarrollar competencias como la búsqueda de bibliografía científica, el pensamiento crítico y la capacidad de síntesis, los cuales son elementos fundamentales para la educación continua.
Como criterio general, la totalidad del personal docente consultado coincidió en la importancia del cumplimiento estricto de ciertas actitudes en el estudiantado, como lo es el respeto, la responsabilidad, los lineamientos éticos y la iniciativa. Estos valores son de gran relevancia, por lo tanto, deben fomentarse durante el proceso de enseñanza a fin de formar profesionales integrales; que no solo manejen los criterios técnico-científicos, sino también los aspectos éticos. Razón por la cual, se propone destinar un apartado de la rúbrica para asegurar el acatamiento de dichas actitudes del alumnado y de esta manera fomentar los valores humanísticos.

Otro aspecto importante que debe considerarse en esta rúbrica, es que las cirugías son demostrativas como está expuesto en los objetivos del curso, por lo cual, no se evalúa en el estudiantado los procedimientos que recién están aprendiendo y que más bien, están analizando y participando como asistentes de cirugía. Por lo tanto, en el rubro de ejecución se evalúan otros parámetros relacionados con la cirugía que aprendieron en cursos previos, como son las normas asépticas y el conocimiento adecuado del instrumental quirúrgico; pues el reiterar los temas vistos en otros cursos fortalece el conocimiento, que será básico cuando se enfrenten a su práctica profesional. Por lo tanto, durante los procedimientos quirúrgicos el alumnado podrá desarrollar las habilidades necesarias para identificar y elegir adecuadamente el instrumental según la intervención quirúrgica que está asistiendo. En cuanto a las normas asépticas, deben aplicarse en todo procedimiento odontológico y más aún, en procedimientos quirúrgicos; donde aumenta el riesgo de contaminación cruzada o infección de la zona quirúrgica. Por lo tanto, se sugiere como parte de la rúbrica, incluir el cumplimiento de las normas asépticas y el conocimiento del instrumental durante el procedimiento quirúrgico.

El apartado final de la rúbrica hace referencia al manejo postquirúrgico, donde 
se evalúa el reconocimiento de la evolución del paciente, así como el proceder según su estado.

Con base en los criterios expuestos se confeccionó la rúbrica de evaluación que aparece en la tabla 1, en la cual se tomaron todas las competencias que debe reunir el estudiantado, para cumplir a satisfacción con los objetivos del curso de Clínica de Exodoncia y Cirugía.

Tabla 1

Rúbrica de evaluación para las presentaciones de caso de la Clínica de Exodoncia y Cirugía

\begin{tabular}{|c|c|c|c|}
\hline $\begin{array}{c}\text { Criterios para la } \\
\text { Presentación de caso }\end{array}$ & $\begin{array}{l}\text { Excelente } \\
(3 \text { ptos })\end{array}$ & $\begin{array}{l}\text { Suficiente } \\
(2 \text { ptos })\end{array}$ & $\begin{array}{l}\text { Deficiente } \\
\quad(1 \mathrm{pto})\end{array}$ \\
\hline $\begin{array}{l}\text { 1. Implicaciones paciente con compromiso sistémico: } \\
\text {-Explica correctamente la patología sistémica } \\
\text {-Describe los métodos diagnósticos de la enfermedad } \\
\text {-Describe los medicamentos y las interacciones } \\
\text { farmacológicas } \\
\text {-Explica el manejo odontológico del paciente }\end{array}$ & $\begin{array}{l}\text { Cumple los } 4 \\
\text { criterios bien }\end{array}$ & $\begin{array}{l}\text { Cumple de } 2 \text { a } 3 \\
\text { criterios bien }\end{array}$ & $\begin{array}{l}\text { Cumple } 1 \\
\text { criterio bien }\end{array}$ \\
\hline $\begin{array}{l}\text { 2. Anatomía de la zona: } \\
\text {-Reconoce estructuras importantes y su implicación }\end{array}$ & $\begin{array}{l}\text { Cumple el } \\
\text { criterio }\end{array}$ & $\begin{array}{l}\text { Solamente } \\
\text { reconoce } \\
\text { estructuras }\end{array}$ & No reconoce \\
\hline $\begin{array}{l}\text { 3. Diagnóstico } \\
\text { En el caso de cirugía de terceras molares, } \\
\text { piezas incluidas: } \\
\text {-Clasifica la tercera molar según Pell Gregory } \\
\text {-Clasifica la tercera molar según Winter } \\
\text {-Realiza un análisis del examen radiográfico }\end{array}$ & $\begin{array}{l}\text { Cumple los } 3 \\
\text { criterios bien }\end{array}$ & $\begin{array}{l}\text { Cumple de } 2 \\
\text { criterios bien }\end{array}$ & $\begin{array}{l}\text { Cumple de } 1 \text { a } 0 \\
\text { criterios bien }\end{array}$ \\
\hline $\begin{array}{l}\text { En el caso de cirugía de tejidos blandos: } \\
\text {-Realiza un diagnóstico diferencial } \\
\text {-Describe las diferentes patologías }\end{array}$ & $\begin{array}{l}\text { Cumple los } 2 \\
\text { criterios bien }\end{array}$ & $\begin{array}{l}\text { Cumple } 1 \\
\text { criterio bien }\end{array}$ & $\begin{array}{l}\text { No cumple los } \\
\text { criterios bien }\end{array}$ \\
\hline $\begin{array}{l}\text { 4. Anestesia } \\
\text {-Menciona los tipos de anestésicos } \\
\text {-Describe la técnica anestésica } \\
\text {-Indica la dosis máxima permitida } \\
\text {-Enumera los componentes químicos del cartucho } \\
\text { de anestesia } \\
\text {-Explica el mecanismo de acción del anestésico }\end{array}$ & $\begin{array}{l}\text { Cumple todos } \\
\text { los criterios } \\
\text { bien }\end{array}$ & $\begin{array}{l}\text { Cumple de } 3 \text { a } 4 \\
\text { criterios bien }\end{array}$ & $\begin{array}{l}\text { Cumple de } 2 \text { a } 0 \\
\text { criterios bien }\end{array}$ \\
\hline $\begin{array}{l}\text { 5. Técnica quirúrgica } \\
\text {-Describe el procedimiento correctamente } \\
\text {-Explica cómo solucionar las urgencias inmediatas } \\
\text {-Menciona las posibles complicaciones y su solución } \\
\text {-Indica el instrumental y materiales a utilizar }\end{array}$ & $\begin{array}{l}\text { Cumple todos } \\
\text { los criterios } \\
\text { bien }\end{array}$ & $\begin{array}{l}\text { Cumple de } 2 \text { a } 3 \\
\text { criterios bien }\end{array}$ & $\begin{array}{l}\text { Cumple de } 2 \text { a } 0 \\
\text { criterios bien }\end{array}$ \\
\hline $\begin{array}{l}\text { 6. Farmacoterapia } \\
\text {-Realiza una terapia farmacológica adecuada } \\
\text {-Menciona la razón por la cual los receta }\end{array}$ & $\begin{array}{l}\text { Cumple todos } \\
\text { los criterios } \\
\text { bien }\end{array}$ & $\begin{array}{l}\text { Receta pero no } \\
\text { menciona por } \\
\text { qué recetó el } \\
\text { fármaco }\end{array}$ & $\begin{array}{l}\text { No cumple con } \\
\text { los criterios }\end{array}$ \\
\hline $\begin{array}{l}\text { 7. Tema teórico asignado } \\
\text {-Buscó referencias bibliográficas pertinentes y de } \\
\text { revistas científicas } \\
\text {-Analiza el tema (Pensamiento crítico) } \\
\text {-Sintetiza y explica el tema adecuadamente }\end{array}$ & $\begin{array}{l}\text { Cumple todos } \\
\text { los criterios } \\
\text { bien }\end{array}$ & $\begin{array}{l}\text { Cumple } 2 \\
\text { criterios bien }\end{array}$ & $\begin{array}{l}\text { Cumple de } 1 \text { a } 0 \\
\text { criterios bien }\end{array}$ \\
\hline
\end{tabular}




\begin{tabular}{|c|c|c|c|}
\hline $\begin{array}{c}\text { Criterios para la } \\
\text { Presentación de caso }\end{array}$ & $\begin{array}{l}\text { Excelente } \\
(3 \text { ptos })\end{array}$ & $\begin{array}{l}\text { Suficiente } \\
\text { (2 ptos) }\end{array}$ & $\begin{array}{l}\text { Deficiente } \\
\quad(1 \mathrm{pto})\end{array}$ \\
\hline $\begin{array}{l}\text { 8. Instrumental, asepsia y farmacoterapia } \\
\text {-Reconoce y toma adecuadamente el instrumental } \\
\text {-Cumple las normas asépticas } \\
\text {-Receta los fármacos correctamente, según } \\
\text { lo discutido }\end{array}$ & $\begin{array}{l}\text { Cumple todos } \\
\text { los criterios } \\
\text { bien }\end{array}$ & $\begin{array}{l}\text { Cumple con } 2 \\
\text { criterios bien }\end{array}$ & $\begin{array}{l}\text { Cumple de } 1 \text { a } 0 \\
\text { criterios bien }\end{array}$ \\
\hline $\begin{array}{l}\text { 9. Actitud } \\
\text {-Se relaciona respetuosamente con el paciente } \\
\text {-Se relaciona respetuosamente con el personal } \\
\text { docente } \\
\text {-Se relaciona respetuosamente con el personal } \\
\text { auxiliar } \\
\text {-Cumple con la ética profesional } \\
\text {-Es responsable en el quehacer clínico } \\
\text {-Demuestra una actitud activa } \\
\text {-Demuestra Iniciativa }\end{array}$ & $\begin{array}{l}\text { Cumple todos } \\
\text { los criterios } \\
\text { bien }\end{array}$ & $\begin{array}{l}\text { Cumple con } 6 \text { a } \\
4 \text { criterios bien }\end{array}$ & $\begin{array}{l}\text { Cumple de } 3 \text { a } 0 \\
\text { criterios bien }\end{array}$ \\
\hline $\begin{array}{l}\text { 10. Criterios de evaluación para el } \\
\text { control postoperatorio }\end{array}$ & $\begin{array}{l}\text { Excelente } \\
\text { (3 ptos) }\end{array}$ & $\begin{array}{l}\text { Suficiente } \\
\text { (2 ptos) }\end{array}$ & $\begin{array}{l}\text { Deficiente } \\
\quad(1 \text { pto })\end{array}$ \\
\hline $\begin{array}{l}\text { - Reconoce la evolución del paciente } \\
\text { - Procede en forma correcta, según el estado } \\
\text { del paciente }\end{array}$ & $\begin{array}{l}\text { Cumple todos } \\
\text { los criterios } \\
\text { bien }\end{array}$ & $\begin{array}{lr}\text { Reconoce } & \text { la } \\
\text { evolución } & \text { pero } \\
\text { desconoce } & \\
\text { proceder } & \end{array}$ & $\begin{array}{l}\text { No cumple con } \\
\text { los criterios }\end{array}$ \\
\hline
\end{tabular}

Fuente: Realizado por la autora.

Se confeccionó una escala para jerarquizar las competencias por evaluar en la rúbrica, según el grado académico demostrado por el estudiantado; tal como propone Tenbrink (1999), se utilizó un número impar de categorías para cada competencia. El extremo positivo fue definido como excelente y se le asignó un puntaje de tres; el extremo opuesto se denominó deficiente y su puntaje fue de uno; quedando como intermedio la categoría de suficiente, equivalente a dos puntos. Este tipo de escala permite evaluar cualitativamente las "presentaciones de caso de cirugía", pues se basa en la descripción verbal de cada categoría según rangos jerarquizados, los cuales permiten calificar al estudiantado según lo demostrado. Aunque, a cada rango se le asigna un puntaje cuantitativo, que en el presente caso fue de positivo a negativo y se le asignó un puntaje a cada uno, respetando un continuum de valores, con diferencias uniformes entre ellas (Tenbrink, 1999).

\section{Conclusiones}

La rúbrica presentada estandariza la evaluación, tomando en consideración los objetivos del curso y las competencias que el personal académico manifestó como parámetros relevantes, que deben considerarse en la evaluación; es importante acotar que se trata de la evaluación hecha por un grupo de 15 docentes, por lo que es fundamental unificar criterios, para que la evaluación sea equitativa, independientemente del profesor o profesora a cargo. Por otro lado, este instrumento de evaluación permite que el personal docente retroalimente al estudiantado según los resultados obtenidos; debido a que la rúbrica le indica al alumnado las áreas en las cuales presenta errores y por lo tanto, la materia que deberá estudiar, para mejorar en próximas "presentaciones de caso de cirugía" e inclusive, le brinda las posibilidades para fortalecer su desarrollo profesional. A su vez, el profesorado podrá analizar las áreas 
que presentan mayores dificultades, con el objetivo de re-plantear y fortalecer actividades académicas que favorezcan el entendimiento de esos temas y con ello mejorar el proceso académico.

También, al desglosar cada uno de los parámetros en indicadores se sopesa el conocimiento del educando, permitiendo una evaluación por actividades individuales que incorpora calificaciones con puntajes intermedios previamente establecidos, en los casos que lo amerite. Debido a que anteriormente no existían puntajes intermedios predeterminados, la calificación tenía un tinte subjetivo y en algunos casos era injusta ya que los criterios de evaluación no eran uniformes entre el equipo docente.

Con respecto al alumnado, esta rúbrica le permite conocer los criterios que se emplean en la evaluación y con ello les motiva a esforzarse para alcanzar la excelencia; inclusive, pueden hacer una autoevaluación del trabajo realizado previo a la presentación frente al docente, de manera que puedan mejorar las áreas débiles. Además, la rúbrica les permite conocer de antemano los criterios que les evaluará el personal académico.

En conclusión, siguiendo los lineamientos planteados en esta rúbrica, que es la segunda propuesta en la Facultad de Odontología, el profesorado no solo se limita a dictar una calificación, sino que se convierte en un orientador, que señala la mejor senda que ha de seguir el educando en el proceso de formación, volcando así su experiencia profesional y de vida, en su quehacer docente diario frente al estudiantado. Con respecto a este, la rúbrica será una guía didáctica, en la que podrá ver, no solo los criterios con que será evaluado, sino que se abrirá al diálogo en ese proceso de aprendizaje, para que el binomio docente-estudiante, sea realmente un ente dinámico de construcción de conocimientos y deje de lado la actitud pasiva de parte del alumnado que solo se limitan a recibir información, que muchas veces aceptan sin procesar y por lo tanto sin comprender, por lo que las dudas se presentan cuando repasa para un examen o peor aún, en una situación real, frente a su paciente. Con esta rúbrica, se promueve una actitud activa del estudiantado, que al conocer de antemano los parámetros que se han clasificado como fundamentales para su evaluación, como se plantea en las "presentaciones de caso de cirugía”, podrá ahondar en su estudio previo, comprenderá mejor la esencia de la actividad y será partícipe de su propio aprendizaje.

\section{Agradecimientos}

Agradezco a los(las) revisores anónimos, por las acertadas sugerencias que permitieron mejorar tanto la redacción como el fundamento teórico de la evaluación. Así mismo, agradezco el apoyo del personal del Departamento de Ciencias Diagnósticas y Quirúrgicas de la Facultad de Odontología y de las autoridades académicas, que hicieron posible esta investigación.

\section{Referencias bibliográficas}

Altbach, P., Reisberg, L. y Rumbley, L. (2009). Trends in global higher education: Tracking an academic revolution. Francia: United Nations Educational, Scientific and Cultural Organization.

Curran, V., Casimiro, L., Banfield,V., Hall, P., Lackie, K., Simmons, B., Tremblay, M., Wagner, S., Oandasan, I. (2009). Research for interprofessional competency- based evaluation. Journal of Interprofessional Care, 23(3), 297-300.

Filloy, C. y Howard, M. (2009). El compromiso de mejoramiento de la Facultad de Odontología de la Universidad de Costa Rica con el Sistema Nacional de Acreditación de la Educación Superior (SINAES), Odovtos, 11, 64-72. 
Foss, H. (2009). Educational evaluation in scandinavian countries: Converging or diverging practices? Scandinavian Journal of Educational Research, 53(1), 71-87.

Hernández, R. y Moreno, S. (2007). La evaluación cualitativa: Una práctica compleja. Educación y Educadores, 10(2), 215-223.

Kan, A. (2007). An alternative method in the new educational program from the point of performance-based assessment: Rubric scoring scales, Educational Sciences: Theory \& Practice, 7 (1), 144-152.

Martínez-Rojas, J.G. (2008). Las rúbricas en la evaluación escolar: Su construcción y su uso. Avances en medición, 6, 129-138.

Morales Vallejo, P. (2010). Ser profesor: Una mirada al alumno. $2^{\mathrm{a}}$ edición. Guatemala: Universidad Rafael Landívar

Maroto, O. (2010). Evaluación de la presentación de caso, Clínica de odontología de restaurativas: Propuesta de una metodología con rúbrica, Revista Electrónica "Actualidades Investigativas en Educación”, 10(1), 1-22.

Reeves, S. y Stanford, B. (2009). Rubrics for the classroom assessment for students and teachers, The Delta Kappa Gamma Bulletin Fall, 24-27.

Santamaría, M.A. (2005). ¿Cómo evaluar aprendizajes en el aula? Costa Rica. Universidad Estatal a Distancia.

Tejada, J. (2005). El trabajo por competencias en el prácticum: Cómo organizarlo y cómo evaluarlo, Revista Electrónica de Investigación Educativa, 7(2), 1-31.

Tenbrink, T.D. (1999). Evaluación guía práctica para profesores (5ta ed.). (Fernández, C., Traductor). España: Narcea.

Valenzuela, J.R. (2004). Evaluación de instituciones educativas. México: Trillas. 